\title{
White Matter Mapping in DT-MRI Using Geometric Flows.
}

\author{
Lisa Jonasson $^{1}$, Patric Hagmann ${ }^{1}$, Xavier Bresson ${ }^{1}$, Reto Meuli ${ }^{2}$, \\ Olivier Cuisenaire ${ }^{1}$ and Jean-Philippe Thiran $^{1}$ \\ 1 Signal Processing Institute (ITS), \\ Swiss Federal Institute of Technology (EPFL), \\ CH-1015 Lausanne, Switzerland \\ \{Lisa. Jonasson, Xavier.Bresson, Patric. Hagmann, \\ Olivier.Cuisenaire, JP. Thiran\}@epfl.ch \\ http://ltswww.epfl.ch/ brain \\ 2 Department of Diagnostic and Interventional Radiology, \\ Lausanne University Hospital (CHUV), \\ CH-1011 Lausanne, Switzerland \\ Reto.Meuli@chuv.hospvd.ch
}

\begin{abstract}
We present a 3D geometric flow designed to evolve in Diffusion Tensor Magnetic Resonance Images(DT-MRI) along fiber tracts by measuring the diffusive similarity between voxels. Therefore we define a front propagation speed that is proportional to the similarity between the tensors lying on the surface and its neighbor in the propagation direction. The method is based on the assumption that successive voxels in a tract have similar diffusion properties. The front propagation is implemented using level set methods by Osher and Sethian [1] to simplify the handling of topology changes and provides an elegant tool for smoothing the segmented tracts. While many methods demand a regularized tensor field, our geometrical flow performs a regularization as it evolves along the fibers. This is done by a curvature dependent smoothing term adapted for thin tubular structures. The purpose of our approach is to get a quantitative measure of the diffusion in segmented fiber tracts. This kind of information can also be used for white matter registration and for surgical planning.
\end{abstract}

\section{Introduction}

Diffusion tensor MRI (DT-MRI) is a relatively new modality that permits noninvasive quantification of the water diffusion in living tissues. The diffusion tensor provides information about both quantity and directions of the main diffusions at a certain point. The water diffusion in the brain is highly affected by the ordered structures of axons, cell membranes and myelin sheaths. The DT becomes highly anisotropic in these fibrous regions and DT-MRI is therefore a useful tool for locating these kinds of structures, providing important information about the brain connectivity, with potential major impact on fundamental neuroscience as well as on clinical practise for a better understanding of many related diseases 
such as multiple sclerosis [2][3], Alzheimer's disease [4] [5], schizophrenia [6][7] and dyslexia [8].

The DT is normally interpreted by calculating its eigenvalues and eigenvectors, the eigenvector corresponding to the highest eigenvalue describes the direction of the principal diffusion and the eigenvalue is a quantitative measure of the diffusion in that direction. Most of the existing methods for tracking fiber bundles rely on this principal diffusion direction to create integral curves describing the fiber paths [9] [10] [11]. Other methods are using a probabilistic approach to explore more of the information contained in the diffusion tensor like for example Hagmann et al. that consider the tensor as a probability distribution [12]. Parker et al. [13] have used level set theory, applied using fast marching methods, to find the connection paths between different brain regions. Campbell et al. [14] have also used level set theory but used it to implement a geometrical flow to track the fiber. They mostly focus on the problem to prevent leakage of the thin tubular structure that represents the fibers, by using flux maximizing flows.

Batchelor et al. [15] are using more of the tensor information by iteratively solving the diffusion equation. The method creates paths that originates from a chosen seed-point, and can be considered as probability measures of a connection. A similar approach is presented by O'Donnell et al. [16] where they find the steady state of the diffusion equation to create a flux vector field. In the same paper they show how the inverse diffusion tensor can define a Riemannian metric that is then used to find geodesic paths that can be interpreted as fiber tracts.

The above methods focus on finding individual fiber paths whereas we have chosen to search for regions corresponding to certain fiber tracts, following the same idea as Tench et al. [17]. For this we use a 3D geometric flow designed to evolve along the fiber tracts by measuring the diffusive similarity between voxels. The front propagation is implemented using level set methods by Osher and Sethian [1]. It simplifies the handling of topology changes and provides an elegant tool for smoothing the segmented tracts. While many methods demand a regularized tensor field, our geometrical flow performs a regularization as it evolves along the fibers. The purpose of our approach is to get a quantitative measure of the diffusion in fiber tracts. This kind of information can also be used for white matter registration and for surgical planning.

\section{Background Theory}

\subsection{Diffusion Tensor Imaging and Tensor Similarity Measures}

Diffusion tensor magnetic resonance imaging(DT-MRI) is a relatively new imaging modality that permits in vivo measures of the self-diffusion of water in living tissues. The tissue structure will affect the Brownian motion of the water molecules which will lead to an anisotropic diffusion. This anisotropic motion can be modelled by an anisotropic Gaussian, that can be parameterized by the diffusion tensor in each voxel [18] to create a 3D field of diffusion tensors. 
The diffusion tensor is a $3 \times 3$ symmetric, semi-positive definite matrix. By diagonalizing the DT we obtain the eigenvalues $\left(\lambda_{1}, \lambda_{2}, \lambda_{3}\right.$ where $\left.\lambda_{1} \geq \lambda_{2} \geq \lambda_{3}\right)$ and the corresponding eigenvectors $\left(\mathbf{e}_{\mathbf{1}}, \mathbf{e}_{\mathbf{2}}, \mathbf{e}_{\mathbf{3}}\right)$. Since the tensor is symmetric and semi-positive definite the eigenvalues will always be positive as long as they are unaffected by noise. The diffusion tensor can then be described in terms of its eigenvalues and eigenvectors.

$$
D=\left(\mathbf{e}_{\mathbf{1}} \mathbf{e}_{\mathbf{2}} \mathbf{e}_{\mathbf{3}}\right)\left(\begin{array}{ccc}
\lambda_{1} & 0 & 0 \\
0 & \lambda_{2} & 0 \\
0 & 0 & \lambda_{3}
\end{array}\right)\left(\mathbf{e}_{\mathbf{1}} \mathbf{e}_{\mathbf{2}} \mathbf{e}_{\mathbf{3}}\right)^{\mathbf{T}} .
$$

The largest eigenvalue and its corresponding eigenvector describes the quantity and direction of the principal diffusion.

Alexander et al.[19,20] have been exploring many similarity measures for tensors to perform elastic matching of diffusion tensor images. These measures do not only take the magnitudes of the diffusivity into account but also the directions. The most common similarity measure between two tensors are the tensor scalar product (TSP). This is a measure of the the overlap between two tensors:

$$
D_{1}: D_{2}=\operatorname{Trace}\left(D_{1} D_{2}\right)=\sum_{j=1}^{3} \sum_{i=1}^{3} \lambda_{1 i} \lambda_{2 j}\left(e_{1 i} e_{2 j}\right)^{2} .
$$

The TSP is often normalized to avoid influence of the relative size of the two tensors. This will emphasize the shape and orientation of the tensor.

$$
\operatorname{NTSP}\left(D_{1}, D_{2}\right)=\frac{D_{1}: D_{2}}{\operatorname{Trace}\left(D_{1}\right) \operatorname{Trace}\left(D_{2}\right)}
$$

This measure is dependent on the shape of the tensor and only a completely anisotropic tensor with diffusion in only one direction compared with itself will sum up to one. In some cases this can be a disadvantage but in our application this will be an advantage since it is the anisotropic regions that are of highest interest.

\subsection{Geometrical Flows and Level Set Implementation}

Geometrical flows and especially curvature- or curve shortening flows are becoming more and more important tools in computer vision. A curvature flow is a curve or surface that evolves at each point along the normal with the velocity depending on the curvature at that point. The theory is well developed for the two dimensional case and even though some of the properties of the $2 \mathrm{D}$ curves, such as the property of shrinking to a point under curvature flow, do not hold in the $3 \mathrm{D}$ case, the main part of the theories remains valid and works well for segmentation of 3D objects.

To use the geometrical flows for image segmentation, the evolution of the curve or surface has to depend on external properties dependent on the image features. A classical speed function to segment gray scale images is based on the gradient of the images and goes to zero when the surface approaches an edge. 
A general flow for a 3D closed surface can be described as:

$$
\frac{\partial S}{\partial t}=(F+\mathbf{H}) \vec{N},
$$

where $F$ is an image based speed function and $\mathbf{H}$ is an intrinsic speed dependent on the curvature of the surface.

To solve this time dependent PDE we use the level set method, introduced by Osher and Sethian [1], where the evolving surface is considered as a constant level set of a function of a higher dimension. By doing this we obtain a numerically stable algorithm that easily handles topology changes of the evolving surface. In our case the function of higher dimension is the signed distance function, $\phi(t)$, of the evolving surface. This makes the evolution of the constant level set coincide with the evolution of $S(t)$. Thus, the evolution of the signed distance function is described by:

$$
\phi_{t}=-(F+\mathbf{H})|\nabla \phi| .
$$

\section{Method}

\subsection{Similarity Based Front Propagation}

As mentioned in the introduction we propose a front propagation method that is based on the assumption that the diffusion is similar between two adjacent voxels within the same tract. To perform the segmentation a small initial surface is placed inside the tract we wish to segment and the surface is then propagated using the similarity measure in (3). The front propagates into a voxel with a speed proportional to the similarity between the diffusion tensor in the voxel and the diffusion tensors in the adjacent voxels lying inside the fiber. The front propagation speed is defined as:

$$
F=\operatorname{mean}\left(\operatorname{NTSP}\left(D_{i}, D_{i-1}\right), \operatorname{NTSP}\left(D_{i}, D_{i-2}\right)\right)
$$

where NTSP is the normalized tensor scalar product as in (3). $D_{i}$ is the current voxel and $D_{i-n}$ are the voxels found by following the normal to the surface $n$ times backwards from the original voxel $i$, see Fig. 1 . The flow does not necessarily evolve in the direction of the diffusion, but the shape of the diffusion tensor is not allowed to differ too much from the local neighborhood inside the fiber. This allows the surface to propagate towards the sides of the fiber tract and thereby segment the whole tract.

\subsection{Regularization}

Due to a high level of noise in the DT-MRI a segmentation based on only the diffusive properties will be very irregular. To smooth the tracts while segmenting them we regularize the flow by adding a curvature dependent speed. Lorigo et al. introduced the use of a curvature definition from codimension 2 flows on surfaces 


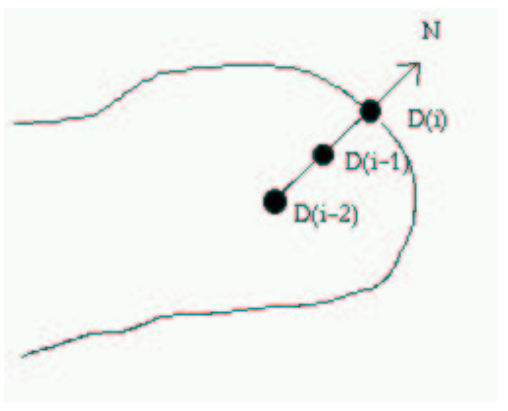

Fig. 1. Choice of adjacent voxels with respect to the normal.

with a thin, tubular structure [21]. Instead of using either mean curvature or gaussian curvature, which will normally destroy the tubular structure, they use the smaller principal curvature which is a combination of both curvatures. The smaller principle curvature, $\kappa$, is given by:

$$
\kappa=H-\sqrt{H^{2}-K},
$$

where $H$ is the mean curvature and $K$ is the gaussian curvature. For the definitions of the mean- and gaussian curvature we refer to [22]. This definition of the curvature will smooth the tubes as if they were open curves in a 3D space, instead of smoothing their tubular form. We will use this definition for our curvature dependent smoothing term.

Our geometric flow now has the form:

$$
\frac{\partial S}{\partial t}=(F+\kappa) \vec{N}
$$

This can easily be implemented with the level set method according to the above theories.

\subsection{Thresholding}

If the speed at one voxel is not equal to zero it will eventually lead to a propagation of the front at that voxel, even though the speed might be very small. To prevent unwanted propagation all speeds inferior to a certain threshold are set to zero. Thresholding is a very abrupt method so it risks to cause discontinuities in the propagation. To avoid this, the Heaviside function defined in [23] is used to get a smoother thresholding.

$$
H_{\epsilon}(x)= \begin{cases}0 & \text { if } x<T-\epsilon \\ \frac{1}{2}\left[1+\frac{x-T}{\epsilon}+\frac{1}{\pi} \sin (\pi(x-T) / \epsilon)\right] & \text { if }|x| \leq \epsilon \\ 1 & \text { if } x>T+\epsilon\end{cases}
$$

where $T$ is the selected threshold. 
The surface evolution is stopped when the propagation speed has been sufficiently small for several succeeding iterations.

\subsection{Implementation}

The method has been implemented in Matlab 6.1 except for the reinitialization of the signed distance function, which has been implemented in $\mathrm{C}$ and compiled with the mex-library, so the function can be called from Matlab.

\section{Validation and Results}

\subsection{Synthetic Tensor Fields}

To test the method synthetic tensor fields have been created. Tensor values for one isotropic and one anisotropic tensor was taken from real data on DT-MRI of the brain of a healthy person. The isotropic tensor was then used as a background for synthetic fibers constructed of rotated anisotropic tensors.

With this method two different 3D tensor fields are constructed, presented in Fig. 2. The images show the largest eigenvector of the tensors at a cut along the z-axis. The first tensor field shows a semicircle to show the ability of following a fiber. The second tensor field simulates a branching fiber.

To make the tensor fields more realistic, noise is added [24]. This is done by making the inverse calculation to obtain the six amplitude images from which the diffusion tensors originally would have been acquired in DT-MRI and then add noise on the amplitude images. The added noise is an approximation the Rician noise [25], [26] as it would be on real MR data. After the noise has been added the tensor images are recreated. The resulting tensor fields can be seen in Fig. 2.

The method was then tested on the synthetic images with different levels of SNR. We have used a SNR of 8, 16 and 32. To start the segmentation a small initial surface is placed somewhere inside the synthetic fiber. Good results have been obtained with several different thresholds between 0.45 and 0.5 . These threshold have then been used on the real MR data. Examples of resulting surfaces can be seen in Fig. 3, these are obtained with a SNR=8. Even though the synthetic tensor fields are very noisy the resulting surfaces are relatively smooth due our regularization that is performed as the surface is evolving.

\subsection{Real DT-MRI}

MRI Data Acquisition. The diffusion tensor images we have used were acquired with a clinical MRI scanner (Magnetom Symphony; Siemens, Erlangen, Germany). The data was produced with a diffusion-weighted single-shot EPI sequence using the standard Siemens Diffusion Tensor Imaging Package for Symphony. We acquired 44 axial slices in a 128 by 128 matrix covering the whole brain of healthy volunteers, from the vertex to the end of the cerebellum. The 


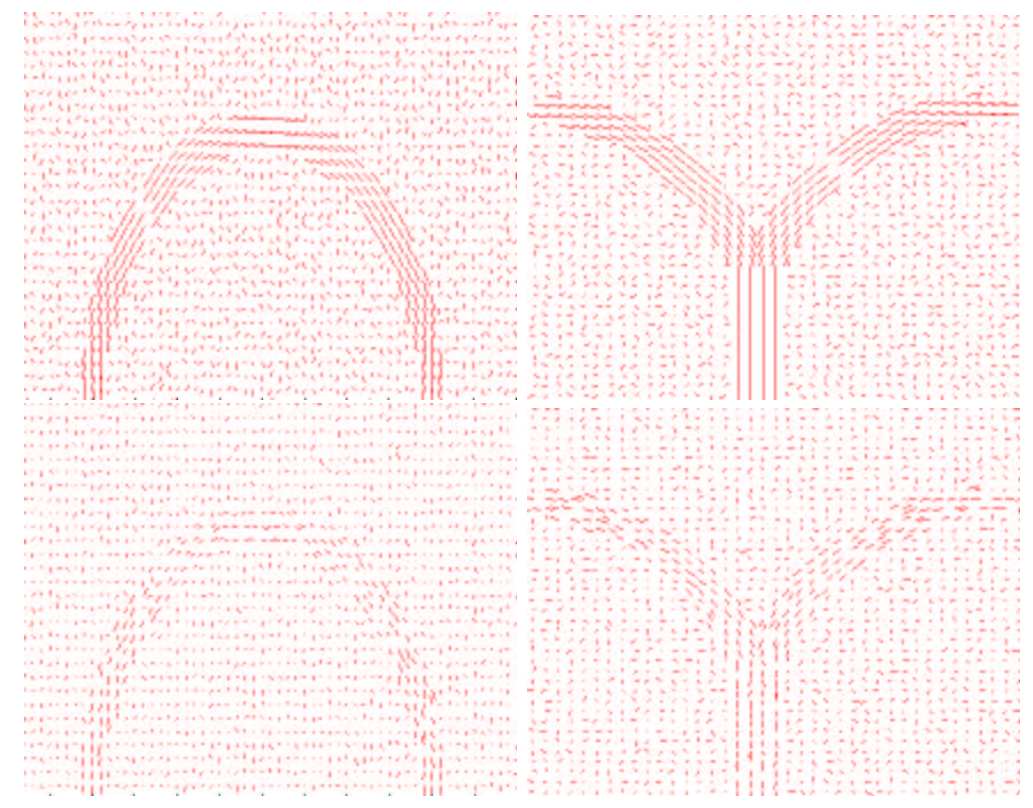

Fig. 2. Synthetic fields: The principal directions of diffusion on a cut along the z-axis. Upper: Before noise is added. Lower: After noise is added.
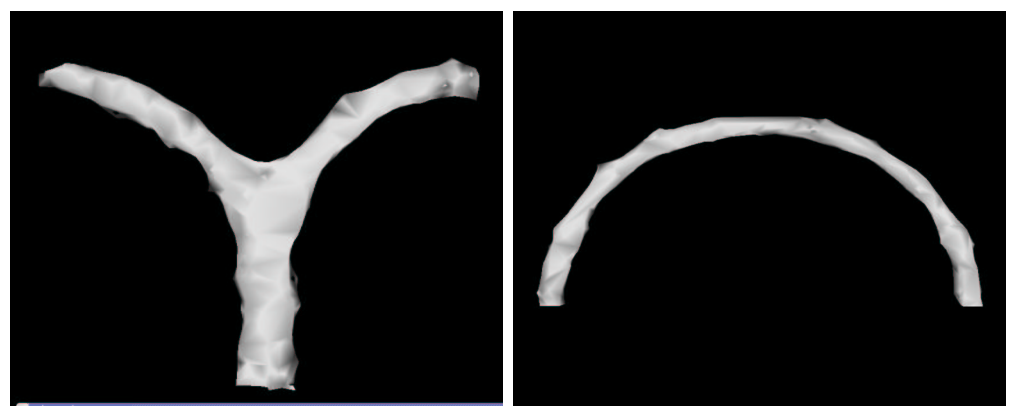

Fig. 3. Example of the resulting segmentation of synthetic vector field with $\mathrm{SNR}=8$ and a threshold of 0.45 . Similar results are obtained for thresholds between 0.45 to 0.5 . 
voxel size was $1.64 \mathrm{~mm}$ by $1.64 \mathrm{~mm}$ with a slice thickness of $3.00 \mathrm{~mm}$ without gap. Timing parameters were a TR of $1000 \mathrm{~s}$ and a TE of 89 s. Diffusion weighting was performed along 6 independent axes and we used a b-value of $1000 \mathrm{~s} / \mathrm{mm}^{2}$ at a maximum gradient field of $30 \mathrm{mT} / \mathrm{m}$. A normalizing image without diffusion weighting was also required. In order to increase the signal to noise ratio the measures were repeated 4 times. An anatomical T1-MP-Range was also performed during the same session. The whole examination lasted about one hour.

Preprocessing of Data. The preprocessing of the data and the geometric flow was carried out in Matlab 6.1. The diffusion tensor was computed for each voxel by linear combination of the log-ratio images according to Basser et al. [27]. The tensors were linearly interpolated component-wise between slices along the z-axis, to obtain a volume with a 3D regular grid of $1.64 \mathrm{~mm}$.

To begin the segmentation an small initial surface is placed inside the fiber tract we wish to segment.

Results The segmentation has been performed on three different DT-MR images. Two of the image acquisitions are from the same person. The results have been validated visually by comparing with post-mortem based neuroanatomical knowledge.

On the synthetic images we saw that several different thresholds have been possible for a good segmentation. On the real MR data the same range of thresholds have been used. Depending on the segmentation we desire the threshold has been varied within the predetermined range. In Fig. 4 the cortico spinal tract is segmented on the three different images. The threshold varies slightly dependent on the image acquisition. In Fig. 5 the corpus callosum has been segmented using two different thresholds. For a stricter threshold a smaller part is segmented but by choosing a lower threshold the surface passes further into the fibers and goes into some of the cortical association bundles.

The cortical association bundles can also be segmented separately as in Fig. 6. For every specific structure the threshold is a little different dependent on the shape of the tract and the anisotropic properties of the diffusion within the tract.

\section{Discussion and Conclusion}

We have presented a new method of segmenting entire fiber tracts by assuming that two adjacent voxels within the same tract has similar diffusion properties. The method manages to segment the larger tracts in the brain. This segmentation can be used as a base for future studies concerning for example quantification of the diffusion in the tracts or for white matter registration.

The segmentation results are sensitive for the choice of parameters. Since there is no objective measure of the exact solution on the brain images is it 



Fig. 4. Segmentation of the cortico spinal tract on three different brain images. The two last rows are images from the same patient but with two different image acquisitions. To obtain a similar segmentation for the three cases the threshold varies slightly dependent on the image acquisition. It is varied between 0.45 to 0.5 . 

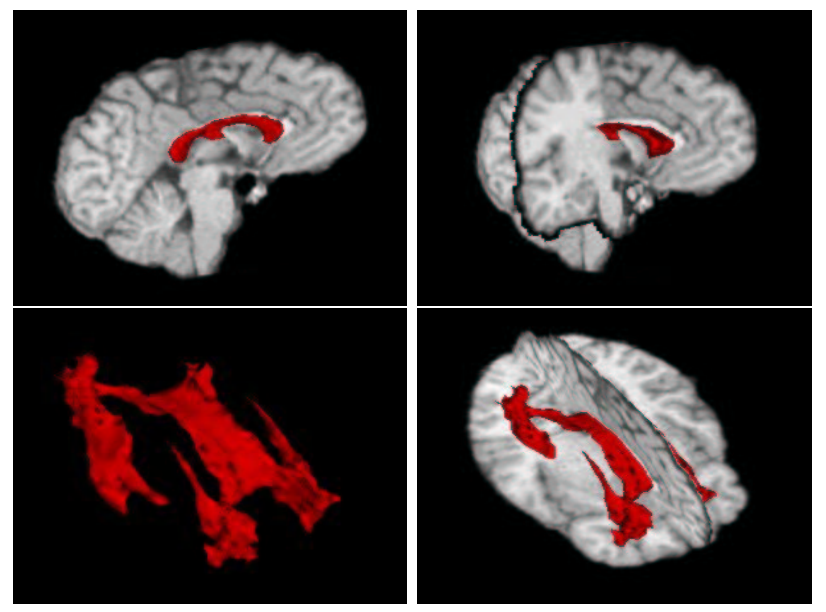

Fig. 5. Results for the similarity based flow on the first test brain. Segmentation of different parts of the corpus callosum for different thresholds. On the two upper images the threshold was set to 0.47 and for the two lower it was set to 0.45 .
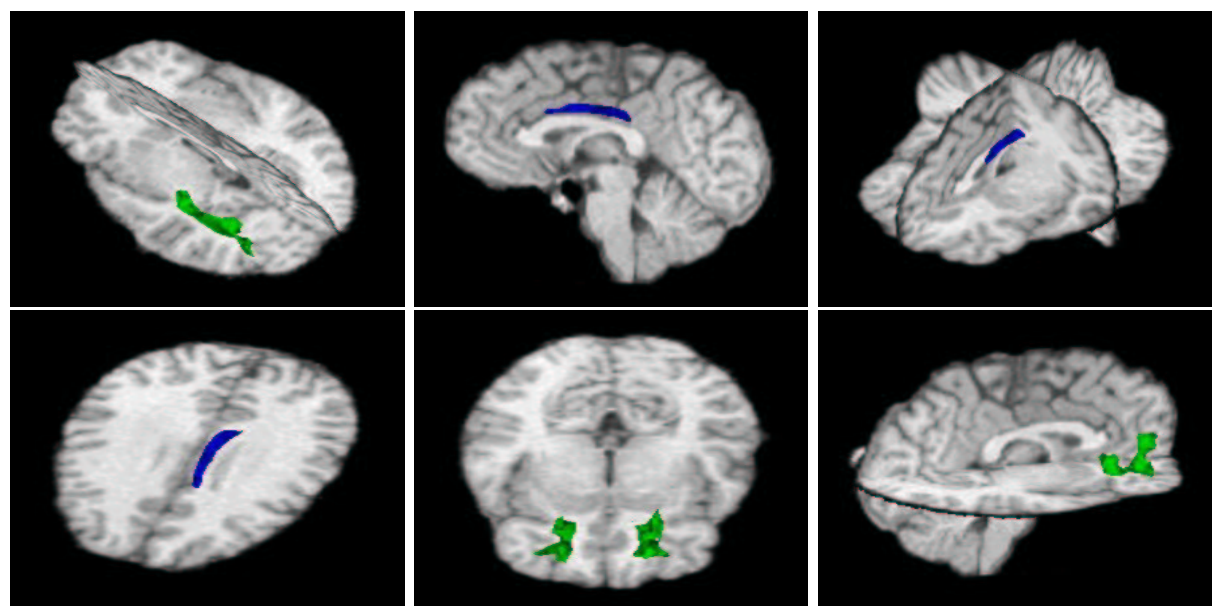

Fig. 6. Results for the similarity based flow on the first test brain. Segmentation of some of the cortical association bundles, threshold set at 0.45 . 
difficult to determine exactly the optimal threshold. This is not necessarily a negative property, an advantage is the flexible segmentation when the choice of threshold determines how far to go in the fibers, see example in Fig. 5.

As mentioned in the introduction most of the existing methods are focusing on following the principal eigenvector of the diffusion tensor. The diffusion tensor contains a lot more information than just the main direction and magnitude of the flow. The other eigenvectors and eigenvalues also contain important data which is often ignored. Only looking at the principal direction also leads to a larger sensitivity to noise since a smaller deviation of the principal direction will lead to an important accumulative error. By exploring more of the tensor information we are creating flows that are less sensitive to noise. The similarity measures is based on the whole tensor and taking all eigenvectors into account.

An important advantage of our approach is the level set implementation. It provides an elegant tool for smoothing the segmented tracts and makes it possible to follow several paths simultaneously and effectively handle branchings and mergings of fibers.

Another advantage of the flow is that we normalize with the total diffusion in each point. This will eliminate the influence of the diffusion strength and make a more correct measure of the common diffusion of the tensors. Calculating the NTSP with adjacent voxels lying inside the propagating surface leads to a regularization of the fiber tract in addition to the regularization performed with the curvature based propagation force.

\section{References}

1. S. Osher and J.A. Sethian. Fronts propagating with curvature-dependent speed: Algorithms based on Hamilton-Jacobi formulations. Journal of Computational Physics, 79:12-49, 1988.

2. M. Filippi, M. Cercignani, M. Inglese, M.A. Horsfield, and G. Comi. Diffusion tensor magnetic resonance imaging in multiple sclerosis. Neurology, 56(3):304-11, 2001.

3. J.A. Maldjian and R.I. Grossman. Future applications of dwi in ms. J Neurol Sci, 186(Suppl 1):S55-7, 2001.

4. S.E. Rose, F. Chen, J.B. Chalk, F.O. Zelaya, W.E. Strugnell, M. Benson, J. Semple, and D.M. Doddrell. Loss of connectivity in alzheimer's disease: an evaluation of white matter tract integrity with colour coded mr diffusion tensor imaging. $J$ Neurol Neurosurg Psychiatry, 69(4):528-30, 2000.

5. M. Bozzali, A. Falini, M. Franceschi, M. Cercignani, M. Zuffi, G. Scotti, G. Comi, and M. Filippi. White matter damage in alzheimer's disease assessed in vivo using diffusion tensor magnetic resonance imaging. J Neurol Neurosurg Psychiatry, $72(6): 742-6,2002$.

6. K.O. Lim, M. Hedehus, M. Moseley, A. de Crespigny, E.V. Sullivan, and A. Pfefferbaum. Compromised white matter tract integrity in schizophrenia inferred from diffusion tensor imaging. Arch Gen Psychiatry, 56(4):367-74, 1999.

7. J. Foong, M. Maier, C.A. Clark, G.J. Barker, D.H. Miller, and M.A. Ron. Neuropathological abnormalities of the corpus callosum in schizophrenia: a diffusion tensor imaging study. J Neurol Neurosurg Psychiatry, 68(2):242-4, 2000. 
8. T. Klingberg, M. Hedehus, E. Temple, T. Salz, J.D. Gabrieli, M.E. Moseley, and R.A. Poldrack. Microstructure of temporo-parietal white matter as a basis for reading ability: evidence from diffusion tensor magnetic resonance imaging. Neuron, 25(2):493-500, 2000.

9. T.E. Conturo, N.F. Lori, T.S. Cull, E. Akbudak, A.Z. Snyder, J.S. Shimony, R.C. McKinstry, H. Burton, and M.E. Raichle. Tracking neuronal fiber pathways in the living human brain. Proc Natl Acad Sci U S A, 96(18):10422-7, 1999.

10. BC. Vemuri, Rao M. Chen, Y., T. McGraw, Z. Wang, and T. Mareci. Fiber tract mapping from diffusion tensor mri. In Proceedings of the IEEE Workshop on Variational and Level Set Methods in Computer Vision, pages 81-88, 2001.

11. P.J. Basser, S. Pajevic, C. Pierpaoli, J. Duda, and A. Aldroubi. In vivo fiber tractography using dt-mri data. Magn Reson Med, 44(4):625-32, 2000.

12. P. Hagmann, J.P. Thiran, L. Jonasson, P. Vandergheynst, S. Clarke, and R. Meuli. Dti mapping of human brain connectivity: Statistical fibre tracking and virtual dissection. NeuroImage, in press, 2003.

13. G.J.M Parker, C.A.M. Wheeler-Kingshott, and G.J. Barker. Distributed anatomical brain connectivity derived from diffusion tensor imaging. In IPMI 2001, pages 106-120, 2001.

14. J. S. W. Campbell, K. Siddiqi, Baba C. Vemuri, and G. B. Pike. A geometric flow for white matter fibre tract reconstruction. In International Symposium On Biomedical Imaging, 2002.

15. P.G. Batchelor, F. Hill, F. Calamante, and D. Atkinson. Study of connectivity in the brain using the full diffusion tensor from mri. In IPMI, pages 121-133, 2001.

16. L. ODonnell, Haker S., and Westin C.F. New approaches to estimation of white matter connectivity in diffusion tensor mri: Elliptic pdes and geodesics in a tensorwarped space. In MICCAI2002, 2002.

17. C.R. Tench, P.S. Morgan, M. Wilson, and L.D. Blumhardt. White matter mapping using diffusion tensor mri. Magn Reson Med, 47(5):967-72, 2002.

18. P.J. Basser, J. Mattiello, and D. Le Bihan. Mr diffusion tensor spectography and imaging. Biophys. J., 66:259-267, 1994.

19. D. Alexander, J. Gee, and R. Bajcsy. Similarity measures for matching diffusion tensor images. In Proceedings BMCV'99, 1999.

20. D. Alexander. Notes on indices of shapes and similarity for diffusion tensors. Technical report, 2001.

21. L.M. Lorigo, O. Faugeras, W.E.L. Grimson, R. Keriven, R. Kikinis, and C.F. Westin. Co-dimension 2 geodesic active contours for mra segmentation. In IPMI 1999, pages 126-139, 1999.

22. JA. Sethian. Level set methods and fast marching methods: Evolving interfaces in computational geometry, fluid mechanics, computer vision, and materials science. 1999.

23. M. Sussman, E. Fatemi, P. Smereka, and S. Osher. An improved level set method for incompressible two-phase flows. Computers and Fluids, 27(5-6):663-680, 1998.

24. J.D. Tournier, F. Calamante, M.D. King, D.G. Gadian, and A. Connelly. Limitations and requirements of diffusion tensor fiber tracking: an assessment using simulations. Magn Reson Med, 47(4):701-8, 2002.

25. H. Gudbjartsson and S. Patz. The rician distribution of noisy mri data. Magn Reson Med, 34:910-914, 1995.

26. RM. Henkelman. Measurement of signal intensities in the prescence of noise. Med. Phys., 12:232-233, 1985.

27. P.J. Basser and C. Pierpaoli. A simplified method to measure the diffusion tensor from seven mr images. Magn Reson Med, 39:928-934, 1998. 